\title{
Publication of study protocols in the CJRT
}

\author{
Justin Sorge, RRT FCSRT MPH
}

Study protocols are an integral part of the research process. Once a viable research plan has been developed by a group of researchers and stakeholders, development and submission of a research protocol to an institutional review or research ethics board is a necessary step in gaining ethics approval. Study protocols outline the research question, sufficient reasoning to conduct research, and detailed methodological plan to be followed. Study protocol review also allows for peer review of the research study plan from both a scientific process lens as well as review of the viability of the research, including funding and budgetary considerations and knowledge dissemination plans. Further, study protocols outline in detail the process researchers plan to fol-

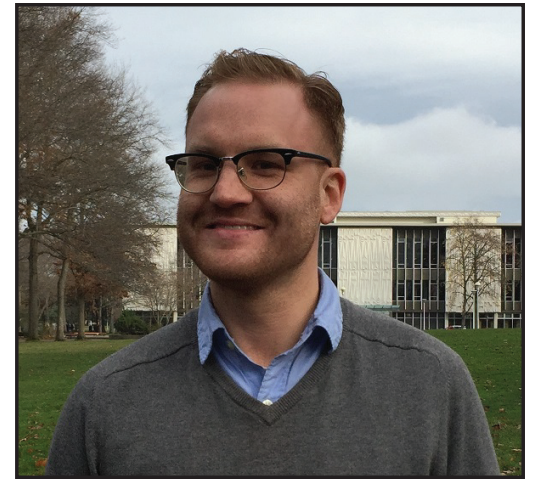

Justin Sorge

concerning research that has not received funding and ethical review will undergo a peer-review process.

We suggest authors use the Preferred Reporting Items for Systematic review and Meta-Analysis Protocols (PRISMA-P) or the Standard Protocol Items: Recommendations for Interventional Trials (SPIRIT) checklists to ensure rapid publication of study protocols $[2,3]$. We look forward to the publication of more protocols in our journal moving forward in efforts to increase transparency in cardiopulmonary health research.

Keep checking back for more interesting articles and taking care of yourselves and those around you!

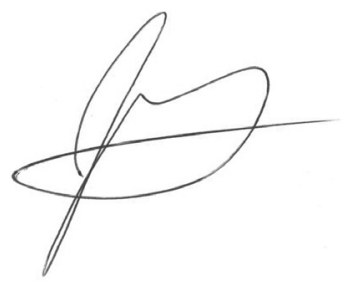

Justin Sorge RRT, FCSRT, MPH, Editor-in-Chief

Of course, unseen and unavoidable events may arise

that cause deviations from the study protocol.

Publishing study protocols can improve the quality of health research. Allowing for an open and transparent review and critique of a protocol can facilitate reproducibility of research, identify publication bias, and is invaluable in assessing risk of bias in systematic review. A recent submission of a research protocol [1] has prompted us at the Canadian Journal of Respiratory Therapy to look into developing guidelines for the publication of research protocols.

Researchers wishing to see their study protocol published in our journal should submit before research has begun-i.e., before participant recruitment or a systematic review literature search has commenced. This is of utmost importance to allow readers and researchers to make assessments of deviations from protocol or selective reporting of findings, for example.

Submitted protocols will be processed on a case-by-case manner to determine if peer-review is necessary. As a general rule, protocols that have received both ethics approval and have been granted funding will have been considered to have already undergone peer-review from both a research ethics and study viability lens. As such, these protocols may only be reviewed by the editor in chief with or without consultation of an editorial board member with subject area knowledge. Protocols

\section{REFERENCES}

1. Zaccagnini M, Bussières A, West A, Boruff J, Thomas A. Features of scholarly practice in health care professionals: a scoping review protocol. Can J Respir Ther 2020;56:38-41. Available from: http://dx.doi. org/10.29390/cjrt-2020-007.

2. Chan AW, Tetzlaff JM, Altman DG, et al. SPIRIT 2013 statement: defining standard protocol items for clinical trials. Ann Intern Med 2013;158(3):200-7. Available from: http://dx.doi.org/10.7326/ 0003-4819-158-3-201302050-00583.

3. Moher D, Shamseer L, Clarke M, et al. Preferred reporting items for systematic review and meta-analysis protocols (PRISMA-P) 2015 statement. Syst Rev 2016;4(1):1. Available from: http://dx.doi.org/1186/2046-4053-4-1. 